\title{
Clinical Characteristics of Intraocular Lens Dislocation in Chinese Han Populations
}

\author{
Qi Fan $\mathbb{D}^{1,2,3,4,5}$ Xiaoyan Han $\mathbb{D}^{1,2,3,4,5}$ Xiangjia Zhu $\mathbb{D}^{1,2,3,4,5}$ Lei Cai $\mathbb{D}^{1,2,3,4,5}$ \\ Xiaodi Qiu $\mathbb{D}^{1},{ }^{1,2,3,4,5}$ Yi Lu $\mathbb{D},,^{1,2,3,4,5}$ and Jin Yang $\mathbb{D}^{1,2,3,4,5}$ \\ ${ }^{1}$ Department of Ophthalmology and the Eye Institute, Eye and Ear, Nose, and Throat Hospital, Fudan University, \\ Shanghai, China \\ ${ }^{2}$ The Key Laboratory of Myopia, Ministry of Health, Shanghai, China \\ ${ }^{3}$ Shanghai Key Laboratory of Visual Impairment and Restoration, Shanghai, China \\ ${ }^{4}$ Key National Health Committee of the Key Laboratory of Myopia, Fudan University, Shanghai, China \\ ${ }^{5}$ The Key Laboratory of Myopia, Chinese Academy of Medical Sciences, Shanghai, China \\ Correspondence should be addressed to Jin Yang; jin_er76@hotmail.com
}

Received 17 March 2020; Accepted 7 April 2020; Published 27 April 2020

Academic Editor: Alessandro Meduri

Copyright (C) 2020 Qi Fan et al. This is an open access article distributed under the Creative Commons Attribution License, which permits unrestricted use, distribution, and reproduction in any medium, provided the original work is properly cited.

Purpose. To investigate the clinical characteristics of patients with intraocular lens (IOL) dislocation after IOL implantation in Chinese Han populations. Methods. The medical records of patients with IOL dislocation were retrospectively reviewed from January 2007 to December 2017, and a total of 312 patients (male: 231, female: 97) (328 eyes) were included in this study. The axial length (AL), IOL power, and the time interval between cataract surgery and IOL dislocation as well as the ocular conditions associated with IOL dislocation were recorded. The IOL dislocation was classified and graded based on its relationship with the capsule and the position of the dislocated IOL. Results. The mean time between original cataract surgery and IOL dislocation was $5.63 \pm 5.13$ years; IOL dislocation occurred in up to $56.1 \%$ (184 eyes) of the eyes within 5 years. Trauma was found in 136 eyes (41.5\%); pars plana vitrectomies were performed in 61 eyes (18.6\%), and high myopia was detected in 108 eyes (32.9\%). A total of 243 eyes $(74.1 \%)$ had out-of-the-bag IOL dislocations, while 85 eyes $(25.9 \%)$ had in-the-bag IOL dislocations. There was a statistically significant difference in the constituent ratio of trauma between in-the-bag dislocation and out-of-the-bag dislocation (Pearson's chi $^{2}=33.3992, P<0.001$ ); ocular blunt traumas were significantly higher in in-the-bag dislocations, while open-globe injuries were significantly higher in out-of-the-bag dislocations. A statistically significant difference was found for the ratio of patients with AL longer than $30 \mathrm{~mm}$ between in-the-bag dislocation and out-of-the-bag dislocation (Pearson's chi ${ }^{2}=9.7355$, $P<0.002)$. Conclusions. In Chinese Han populations, the most common IOL dislocation is out-of-the-bag dislocation; the most common risk factors were trauma, long axial length, and eyes undergoing pars plana vitrectomy; a minimum follow-up of 5 years is suggested for IOL dislocation-predisposed eyes undergoing cataract surgery.

\section{Introduction}

Dislocation of an intraocular lens (IOL) is uncommon but is one of the most serious complications following cataract surgery [1]. It is a cause of blindness throughout the world, with an incidence of $0.05-3 \%$ [2-4] and a cumulative risk of $0.1 \%$ after 10 years and $1.7 \%$ after 25 years following cataract surgery [5]. With recent developments in surgical techniques and devices, cataract surgery has now provided a higher safety for patients, though it does not parallel a declined frequency of IOL dislocation due to the increasing number of cataract surgeries [6].

The causes of IOL dislocation include the absence of adequate capsular or zonular support. Previously reported factors associated with zonular weakness or dehiscence and capsular insufficiency were indicated in eyes with pseudoexfoliation syndrome [7], uveitis [8], retinitis pigmentosa [9], previous vitreoretinal surgery [10], increased axial length [6], Marfan syndrome, and trauma [11]. Detailed information 
TABLE 1: Baseline clinical characteristics.

\begin{tabular}{lcr}
\hline Baseline clinical characteristics & Mean \pm SD & Range \\
\hline Age & $48.10 \pm 20.17$ years & 2 to 85 years \\
Duration of IOL dislocation & $5.63 \pm 5.13$ years & 1 day to 23.39 years \\
Time between IOL dislocation and surgical intervention & $0.53 \pm 1.40$ years & 1 day to 15.39 years \\
\hline Axial length & $25.65 \pm 3.31 \mathrm{~mm}$ & 17.74 to $36.69 \mathrm{~mm}$ \\
$\quad$ In-the-bag dislocation & $26.51 \pm 3.78 \mathrm{~mm}$ & 20.86 to $35.86 \mathrm{~mm}$ \\
Out-of-the-bag dislocation & $25.34 \pm 3.07 \mathrm{~mm}$ & 17.74 to $36.69 \mathrm{~mm}$ \\
\hline
\end{tabular}

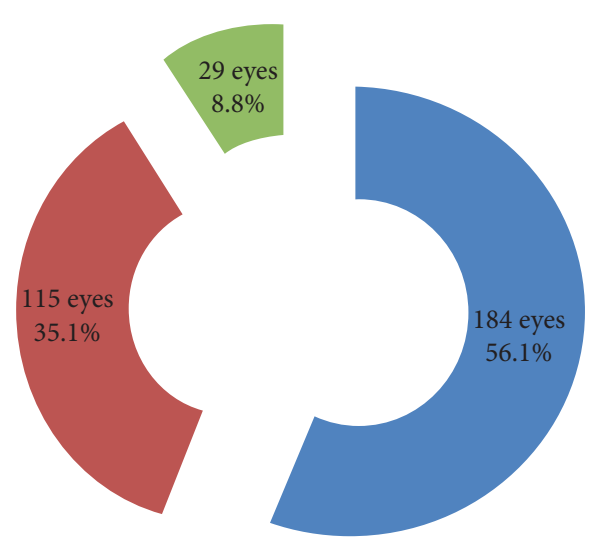

Within 5 years 5 years to 10 years 10 years or more

(a)

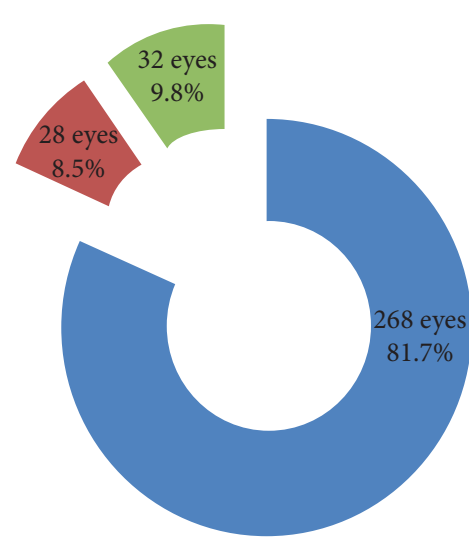

Within 6 months

6 months to 1 years

1 year or more

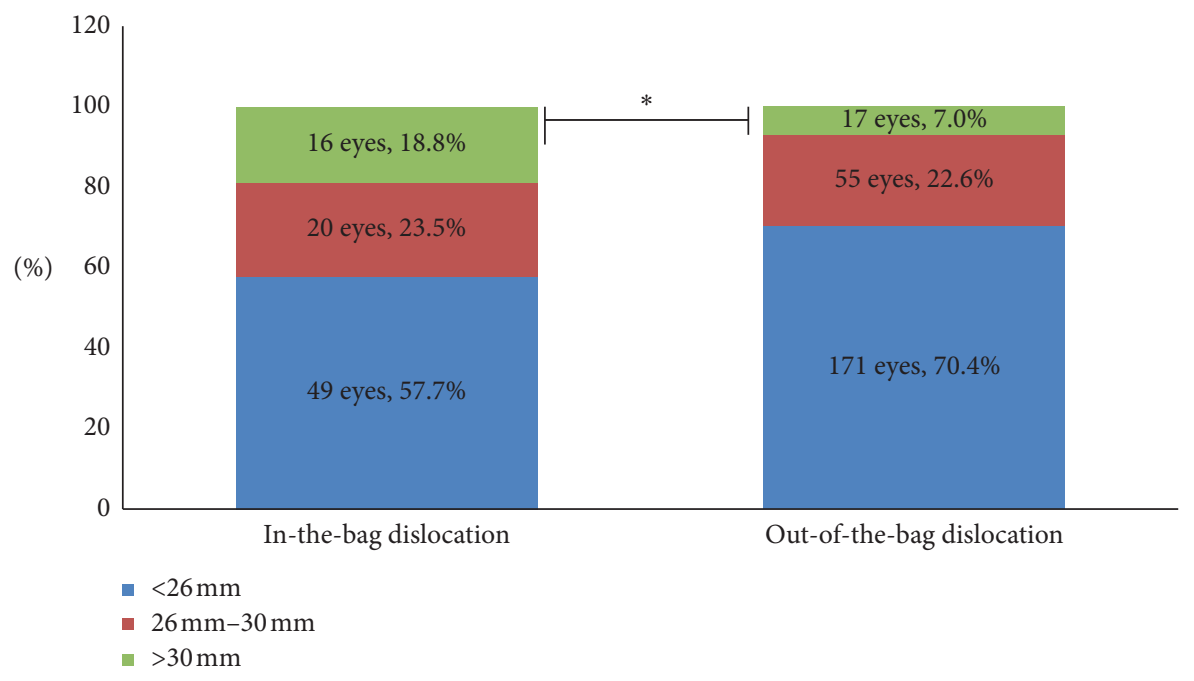

(c)

FIgURE 1: The proportion ratios of time from cataract surgery to IOL dislocation (a), from IOL dislocation to surgical intervention (b), and axial length (c). * Statistically significant differences between in-the-bag dislocation and out-of-the-bag dislocation were found for the ratio of patients with AL longer than $30 \mathrm{~mm}$.

on the clinical characteristics of IOL dislocation has been reported in populations such as American, German, Australian, and Swedish populations, among others. However, to the best of our knowledge, little is known about the risk factors for IOL dislocation in Chinese Han populations.

In this study, a retrospective chart review of consecutive patients with IOL dislocation was performed, and the clinical characteristics of IOL dislocation patients in Chinese Han populations were demonstrated and analyzed.

\section{Materials and Methods}

We retrospectively reviewed all charts of subjects with IOL dislocation after IOL implantation surgery between January 
2007 and December 2017 in Shanghai, and those who required further surgical intervention were included. This study adhered to the Declaration of Helsinki and was approved by the Ethics Committee of the Eye \& ENT Hospital of Fudan University.

Basic information, such as age and sex, axial length (AL), intraocular pressure (IOP), corneal endothelial cell density, corneal astigmatism, time for cataract surgery, time between IOL dislocation and surgical intervention, duration of IOL dislocation, and surgical choices for IOL dislocation, was acquired and demonstrated. Associated ocular conditions when the patients presented to the clinic were also investigated.

2.1. Classification of IOL Dislocation. Several standards for classifying IOL dislocation have been established [12-15].

Based on whether or not the dislocated IOL is in the capsule, it can be classified into two categories: in-the-bag dislocation and out-of-the-bag dislocation; it can also be classified as dislocation in the anterior chamber, in the posterior chamber and/or in the anterior vitreous cavity, and in the deep vitreous cavity, relying on the sites of dislocated IOL. For those whose IOL dislocated in the posterior chamber and/or the anterior vitreous cavity, the sites of dislocated IOL were further described as superior, superonasal, nasal, inferonasal, inferior, inferotemporal, and superotemporal, and the severities were graded as mild, moderate, and severe.

2.2. Statistical Analysis. SPSS software (SPSS, Inc.) was used for the statistical analysis. Continuous variables are presented as means \pm standard deviations. Discrete variables were presented as percentages. Fisher's exact test and chisquared test were used to analyze the discrete variables. Any differences with $P$ values less than 0.05 were considered statistically significant.

\section{Results}

3.1. General Clinical Characteristics. A total of 312 patients (male: 231, female: 97) (328 eyes, 16 patients had bilateral IOL dislocations) were included in this study, with a mean age of $48.10 \pm 20.17$ years, ranging from 2 to 85 years (Table 1). The included patients had an average time from cataract surgery to the onset of IOL dislocation of $5.63 \pm 5.13$ years ( 1 day to 23.39 years, Table 1 ), with $56.1 \%$ (184 eyes) occurring within 5 years, $35.1 \%$ (115 eyes) between 5 and 10 years, and $8.8 \%$ (29 eyes) in more than 10 years (Figure $1(\mathrm{a})$ ). The mean time to surgical intervention was $0.53 \pm 1.40$ years (1 day to 15.39 years), with $81.7 \%$ (268 eyes) of the patients within 6 months, 8.5\% (28 eyes) between 6 months and 12 months, and $9.8 \%$ (32 eyes) in more than one year (Figure 1(b)).

The mean AL was $25.65 \pm 3.31 \mathrm{~mm}$ (17.74 to $36.69 \mathrm{~mm}$ ). For in-the-bag dislocations, the mean AL was $26.51 \pm 3.78 \mathrm{~mm}$ ( 20.86 to $35.86 \mathrm{~mm}$ ), with $18.8 \%$ (16 eyes) of patients having an AL longer than $30 \mathrm{~mm}, 23.5 \%$ (20 eyes) between $26.0 \mathrm{~mm}$ and $30.0 \mathrm{~mm}$, and 57.6\% (49 eyes) shorter
TABLE 2: Associated ocular conditions with IOL dislocation.

\begin{tabular}{lc}
\hline Associated ocular conditions & Eyes $(n / 328)$ \\
\hline Trauma & $136(41.5 \%)$ \\
Open-globe injuries & $95(29.0 \%)$ \\
Before primary IOL implantation & $93(28.4 \%)$ \\
After primary IOL implantation & $2(0.6 \%)$ \\
Ocular blunt trauma & $41(12.5 \%)$ \\
Before primary IOL implantation & $6(1.8 \%)$ \\
After primary IOL implantation & $35(10.7 \%)$ \\
\hline Ocular diseases & $172(52.4 \%)$ \\
High myopia & $108(32.9 \%)$ \\
Lens subluxation & $32(9.8 \%)$ \\
Retinitis pigmentosa & $15(4.6 \%)$ \\
Chronic uveitis & $6(1.8 \%)$ \\
Glaucoma & $5(1.5 \%)$ \\
Iris cyst & $4(1.2 \%)$ \\
Ocular siderosis & $2(0.6 \%)$ \\
\hline Ocular surgeries & $108(32.9 \%)$ \\
Pars plana vitrectomy & $61(18.6 \%)$ \\
Congenital cataract surgery & $30(9.1 \%)$ \\
Trabeculectomy & $6(1.8 \%)$ \\
Strabismus correction & $6(1.8 \%)$ \\
Scleral buckling & $3(0.9 \%)$ \\
Penetration keratoplasty & $2(0.6 \%)$ \\
\hline Capsule rupture & $42(12.8 \%)$ \\
Nd:YAG laser capsulotomy & $8(2.4 \%)$ \\
Capsular contraction syndrome & $21(6.4 \%)$ \\
\hline
\end{tabular}

than $26 \mathrm{~mm}$. For out-of-the-bag dislocations, the mean AL was $25.34 \pm 3.07 \mathrm{~mm}$ (17.74 to $36.69 \mathrm{~mm}$ ), with $7.0 \%$ (17 eyes) of patients having an AL longer than $30 \mathrm{~mm}, 22.6 \%$ (55 eyes) between $26.0 \mathrm{~mm}$ and $30.0 \mathrm{~mm}$, and $70.4 \%$ (171 eyes) shorter than $26 \mathrm{~mm}$ (Figure 1(c)). Statistically significant differences between in-the-bag dislocation and out-of-thebag dislocation were found for the ratio of patients with $\mathrm{AL}$ longer than $30 \mathrm{~mm}$ (Pearson's $\mathrm{chi}^{2}=9.7355, \quad P$ value $=0.002$ ).

3.2. Associated Ocular Conditions. Associated ocular conditions with IOL dislocation are shown in Table 2. A total of 168 eyes $(51.2 \%)$ had more than one associated ocular condition.

A total of 136 eyes (41.5\%) were associated with trauma. Of these, 95 eyes (29.0\%) were open-globe injuries, and 41 eyes $(12.5 \%)$ were ocular blunt trauma. A statistically significant difference between the constituent ratio of trauma before and after primary IOL implantation was found (Pearson's $\mathrm{chi}^{2}=100.25, P$ value $<0.001$ ). The ratio of openglobe injuries was significantly higher before primary IOL implantation than after primary IOL implantation, while ocular blunt trauma was significantly higher after primary IOL implantation than before primary IOL implantation.

A total of 172 eyes (52.4\%) had ocular diseases (Table 2). High myopia was detected in 108 eyes (32.9\%) (AL was longer than $26.00 \mathrm{~mm}$ ); preexisting lens subluxation before original cataract surgery appeared in 32 eyes (9.8\%). Other associated ocular diseases were as follows: retinitis pigmentosa (15 eyes/4.6\%), chronic uveitis (6 eyes/1.8\%), 
TABLE 3: Distributions of IOL dislocation based on classification systems.

\begin{tabular}{lc}
\hline Classifications of IOL dislocation & Eyes $(n / 328)$ \\
\hline Relationship with capsule & $85(25.9 \%)$ \\
In-the-bag dislocations & $243(74.1 \%)$ \\
Out-of-the-bag dislocations & $39(11.9 \%)$ \\
\hline IOL dislocation position & $184(56.1 \%)$ \\
In the anterior chamber & $105(32.0 \%)$ \\
In the posterior chamber and/or the anterior vitreous cavity & \\
In deep vitreous cavity/vitreous completely & \\
\hline
\end{tabular}

glaucoma (5 eyes/1.5\%), iris cyst (4 eyes/1.2\%), and ocular siderosis (2 eyes $/ 0.6 \%$ ).

A total of 108 eyes (32.9\%) had ocular surgeries conditions (Table 2). Pars plana vitrectomy was performed in 61 eyes $(18.6 \%)$, and causes of vitrectomy included retinal detachment (22 eyes/36.1\%), traumatic vitreoretinopathy (19 eyes/31.1\%), intraocular foreign body (10 eyes/16.4\%), macular hole (5 eyes/8.2\%), diabetic retinopathy (3 eyes/ $4.9 \%$ ), and endophthalmitis ( 2 eyes/3.3\%). A total of 30 eyes (9.1\%) underwent surgery for congenital cataract. Other associated surgeries were as follows: trabeculectomy (6 eyes/ $1.8 \%)$, strabismus correction (6 eyes/1.8\%), scleral buckling (3 eyes/0.9\%), and penetration keratoplasty (2 eyes/0.6\%).

A total of 42 eyes $(12.8 \%)$ had a history of capsule rupture during cataract surgery; 8 eyes $(2.4 \%)$ received $\mathrm{Nd}: \mathrm{YAG}$ laser capsulotomy, and 21 eyes $(6.4 \%)$ had capsular contraction syndrome.

3.3. Dislocation Sites and Grading. Detailed information on IOL dislocation classified by the aforementioned classification standard is provided in Table 3.

A total of 85 eyes $(25.9 \%)$ were seen with in-the-bag dislocations (Figure $2(\mathrm{a})$ ), and 243 eyes (74.1\%) had out-ofthe-bag dislocations (Figure 2(b)). IOL dislocation sites were as follows: the intraocular lens was dislocated into the anterior chamber in 39 eyes (11.9\%) (Figure 2(c)), into the posterior chamber and/or the anterior vitreous cavity in 184 eyes $(56.1 \%$ ) (Figure $2(\mathrm{~d})$ ), and completely into the vitreous in 105 eyes $(32.0 \%)$ (Figures 2(e) and 2(f)). The major dislocation sites were inferior in 121 eyes (65.8\%) (Table 4).

3.4. In-the-Bag Dislocations versus Out-of-the-Bag Dislocations. Associated ocular conditions with in-the-bag and out-of-the-bag dislocations are shown in Table 5.

The most common associated ocular conditions with inthe-bag dislocations were high myopia in 36 eyes $(42.4 \%)$, trauma in 26 eyes (30.6\%), and retinitis pigmentosa in 13 eyes $(15.3 \%)$, while the most common associated ocular conditions with out-of-the-bag dislocations were trauma in 110 eyes (45.3\%) (especially open-globe injuries), high myopia in 72 eyes $(29.6 \%)$, and pars plana vitrectomy in 50 eyes $(20.6 \%)$. There was a statistically significant difference between in-the-bag dislocation and out-of-the-bag dislocation on the constituent ratio of trauma (Pearson's $\left.\mathrm{chi}^{2}=33.3992, P<0.001\right)$, and ocular blunt traumas were significantly higher in in-the-bag dislocations, while open- globe injuries were significantly higher in out-of-the-bag dislocations.

3.5. Surgical Procedures for IOL Dislocation. Surgical techniques to treat IOL dislocation included IOL exchange, reposition, refixation, and explantation (Table 6). A total of 19 eyes (5.8\%) suffered from two instances of IOL repositioning. IOL exchange and refixation were the most common surgical procedures, accounting for 39.9\% (131 eyes) and $25.9 \%$ ( 85 eyes), respectively. Sutured scleral fixation is most commonly used for both IOL exchange (98 eyes/ $29.9 \%$ ) and refixation (78 eyes/23.8\%).

\section{Discussion}

As cataract patients are increasing with the aging of the population, IOL dislocation is an unusual but not rare complication following cataract surgery, even with noted progress in surgical techniques and devices. According to this study, the predisposing factors may differ due to ethnic and geographical differences. To the best of our knowledge, the characteristics of IOL dislocation in the Chinese Han population are seldom reported. Due to the surgical difficulty involved in IOL dislocation, patients in eastern China are concentrated in Shanghai to deal with IOL dislocation. As a tertiary referral clinic area and one of the most important ophthalmic centers in China, we have relatively easy access to a larger amount of data relating to the IOL dislocation, based on which its clinical characteristics are provided in this study so that they may further assist in managing this complication in practice.

Contrary to the previous reports, the majority of which have focused on in-the-bag dislocation, our study has demonstrated the clinical characteristics of both in-the-bag and out-of-the-bag dislocation. In light of other ethnic studies [3], pseudoexfoliation was referred to as the most common risk factor predisposing IOL to dislocate, followed by other ocular conditions, such as uveitis, trauma, vitrectomy, and increased axial length, which disagrees with the findings in our study that the most common risk factors were trauma, high myopia, and eyes undergoing pars plana vitrectomy in a Chinese population. This finding may be related to the high incidence of high myopia and the lack of labor protection in China.

In this study, the mean time interval between primary cataract surgery and surgical intervention for IOL dislocation was 5.63 years, shorter than the times reported in previous studies (8.04 years [13] and 8.5 years [15]). The 


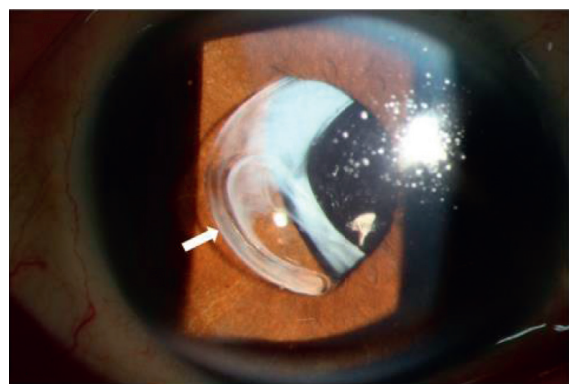

(a)

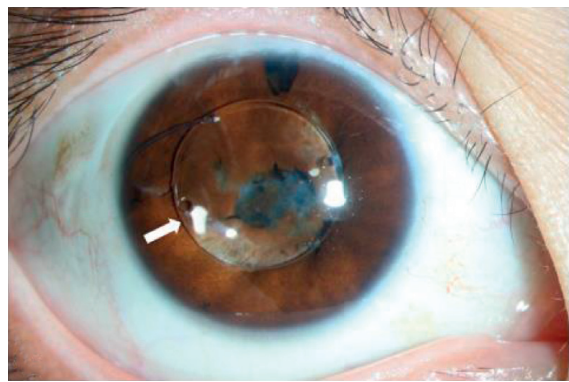

(c)

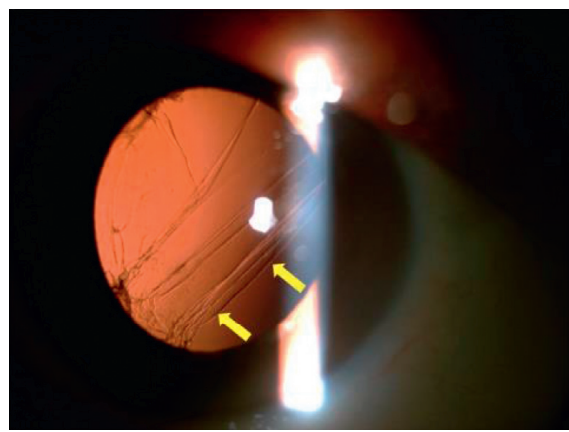

(e)

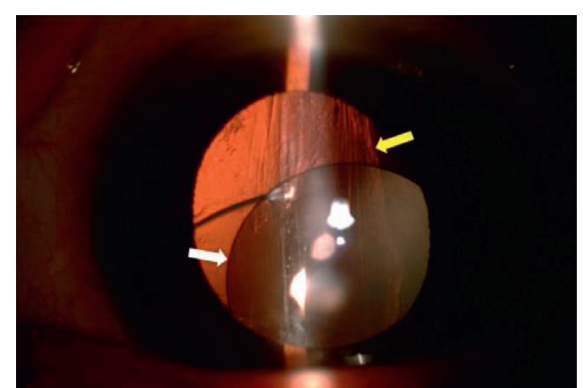

(b)

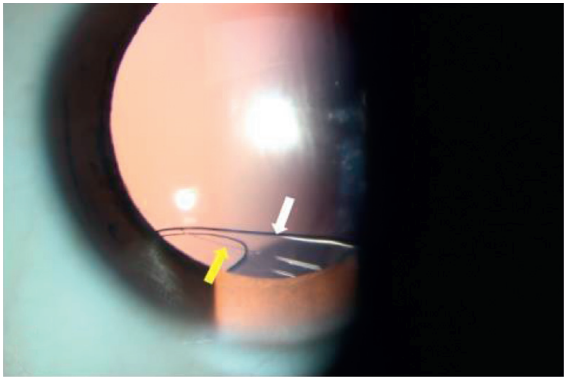

(d)

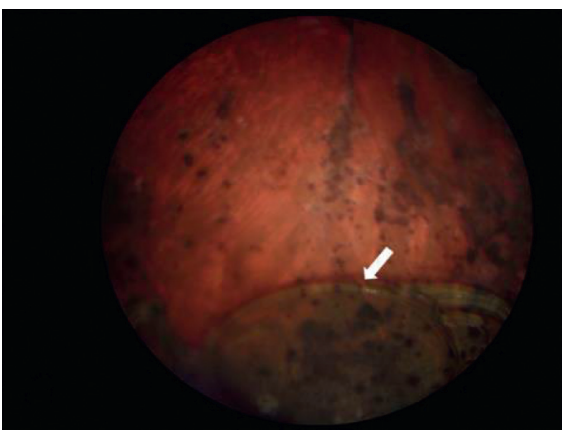

(f)

FIgURE 2: Representative photographs of IOL dislocation. (a) Capsular bag complex dislocation; (b) out-of-the-bag dislocation; (c) prolapse into the anterior chamber; (d) prolapse into the anterior vitreous cavity; (e) and (f) (e) presents the ruptured capsule and absence of IOL, while (f) presents IOL dislocated completely into the vitreous. White arrows show dislocated IOL, while yellow arrows require a ruptured capsule.

reason for this difference may be related to the inclusion of patients with trauma and out-of-the-bag IOL dislocation in this study. As IOL dislocation occurred in more than half of the eyes within 5 years and in less than $9 \%$ of the eyes in excess of 10 years, a minimum follow-up of 5-10 years is needed for the patients with IOL dislocation risk factors, even as the primary cataract surgery is uneventful.

The mean age of IOL dislocation patients was $48.10 \pm 20.17$ years, and $70.4 \%$ were male in this study. The majority of the patients were young- and middle-aged males, which is in accord with the findings in our study, in which trauma is listed as the first common risk factor for IOL dislocation. China is the world's largest population, and young- and middle-aged men are the most engaged in heavy labor work that lacks occupational protection, thus representing a significant trauma exposure risk. Blunt force after primary IOL implantation could result in zonular rupture and dialysis and often leads to in-the-bag IOL dislocations,
TABLE 4: Sites and grades of IOL dislocation.

\begin{tabular}{lc}
\hline Sites and grades of dislocated IOL & Eyes $(n / 184)$ \\
\hline Dislocation grades & \\
Mild & $17(9.2 \%)$ \\
Moderate & $85(46.2 \%)$ \\
Severe & $82(44.6 \%)$ \\
\hline Dislocation sites & \\
Superior & $3(1.6 \%)$ \\
Superonasal & $5(2.7 \%)$ \\
Nasal & $7(3.8 \%)$ \\
Inferonasal & $25(13.6 \%)$ \\
Inferior & $121(65.8 \%)$ \\
Inferotemporal & $18(9.8 \%)$ \\
Superotemporal & $5(2.7 \%)$ \\
\hline
\end{tabular}

while open-globe injuries before primary IOL implantation often lead to out-of-the-bag dislocations, with capsule rupture or capsule incompleteness. 
TABLE 5: Ocular conditions associated with in-the-bag dislocations and out-of-the-bag dislocations.

\begin{tabular}{lcc}
\hline Associated ocular conditions & In-the-bag dislocation (eyes/85) & Out-of-the-bag dislocation (eyes/243) \\
\hline Trauma & $26(30.6 \%)$ & $110(45.3 \%)$ \\
Ocular blunt trauma & $20(23.5 \%)$ & $21(8.6 \%)$ \\
Open-globe injuries & $6(7.1 \%)$ & $89(36.6 \%)$ \\
High myopia & $36(42.4 \%)$ & $72(29.6 \%)$ \\
Lens subluxation & $7(8.2 \%)$ & $25(10.3 \%)$ \\
Retinitis pigmentosa & $13(15.3 \%)$ & $2(0.8 \%)$ \\
Chronic uveitis & $6(7.1 \%)$ & $0(0 \%)$ \\
Glaucoma & $2(2.4 \%)$ & $3(1.2 \%)$ \\
Iris cyst & $0(0 \%)$ & $4(1.6 \%)$ \\
Ocular siderosis & $1(1.2 \%)$ & $1(0.4 \%)$ \\
Pars plana vitrectomy & $11(12.9 \%)$ & $50(20.6 \%)$ \\
Congenital cataract surgery & $2(2.4 \%)$ & $28(11.5 \%)$ \\
Strabismus correction & $1(1.2 \%)$ & $5(2.1 \%)$ \\
Trabeculectomy & $2(2.4 \%)$ & $4(1.6 \%)$ \\
Scleral buckling & $1(1.2 \%)$ & $2(0.8 \%)$ \\
Penetration keratoplasty & $1(1.2 \%)$ & $1(0.4 \%)$ \\
Capsule rupture & $1(1.2 \%)$ & $41(16.9 \%)$ \\
Nd:YAG laser capsulotomy & $0(0 \%)$ & $8(3.3 \%)$ \\
\hline
\end{tabular}

High myopia was another one of the most common risk factors associated with IOL dislocation in this study. At the same time, we found that AL longer than $30 \mathrm{~mm}$ was prone to result in IOL in-the-bag dislocation than out-of-the-bag dislocation, for in-the-bag dislocation patients with AL longer than $30 \mathrm{~mm}$ were significantly higher than out-ofthe-bag dislocation patients. Part of the reason may be the variability in ethnicity, whereas high myopia has a higher prevalence than pseudoexfoliation in China, which resulted in zonule weaknesses. Longer axial length of the eyeball causes 360-degree stretching and dehiscence of zonular fibers, which fails to adequately resist the tension of the anterior capsule contraction and results in progressive capsular contraction or the zonular dialysis. Twenty-one eyes had anterior capsule contraction. And the most common causes, except for high myopia, are retinitis pigmentosa, chronic uveitis, glaucoma, and others.

Pars plana vitrectomy is the most common surgery associated with IOL dislocation. The most common causes of vitrectomy include retinal detachment (36.1\%) and traumatic vitreoretinopathy $(31.1 \%)$. Vitrectomy for retinal detachment is more likely to cause IOL dislocation than vitrectomy for macular disease $36.1 \%$ retinal detachment versus $8.2 \%$ macular hole). Part of the reason may be that aggressive peripheral vitrectomy with scleral depression during the vitrectomy for retinal detachment may damage the posterior zonular fibers. Traumatic vitreoretinopathy is often associated with anterior or posterior capsular rupture. Also before 2011, pars plana vitrectomy used a 20-gauge vitrectomy system with a lower vitrector cutting rate $(20 \mathrm{G}$ vitrectomy $800-2500$ cuts per minute versus $23 \mathrm{G}$ vitrectomy 2500-7500 cuts per minute) and a larger diameter of vitrectomy probe (20G vitrectomy probe $1.0 \mathrm{~mm}$ versus $23 \mathrm{G}$ vitrectomy probe $0.5 \mathrm{~mm}$ ) than a 23-gauge vitrectomy system, which is more likely to damage posterior zonular fibers and the posterior lens capsule. Additionally, damage to the posterior lens capsule may cause traumatic injury of the capsule integrity and, in turn, was linked to a larger
TABLE 6: Surgical techniques to correct IOL dislocation.

\begin{tabular}{lc}
\hline Surgical techniques & Eyes $(n / 328)$ \\
\hline Exchange & $131(39.9 \%)$ \\
Sutured scleral fixation & $98(29.9 \%)$ \\
Iris-claw IOL & $12(3.7 \%)$ \\
Positioning in ciliary sulcus & $21(6.4 \%)$ \\
\hline Reposition & $30(9.1 \%)$ \\
Ciliary sulcus reposition & $30(9.1 \%)$ \\
\hline Refixation & $85(25.9 \%)$ \\
Sutured scleral refixation & $78(23.8 \%)$ \\
Sutureless intrascleral refixation & $7(2.1 \%)$ \\
\hline Explantation & $82(25 \%)$ \\
Simple explantation & $34(10.4 \%)$ \\
Explantation plus anterior vitrectomy & $22(6.7 \%)$ \\
Explantation plus pars plana vitrectomy & $26(7.9 \%)$ \\
\hline
\end{tabular}

percentage of out-of-the-bag dislocations in the present study. A total of 30 eyes with IOL dislocation had previously undergone surgeries for congenital cataract in this study. Because of inadequate primary posterior capsulotomy and/ or anterior vitrectomy during the primary IOL implantation, the anterior vitreous face formed a scaffold for the proliferation of lens epithelial cells, which may contribute to IOL shift forward and result in IOL anterior displacement.

The most common surgical techniques to treat IOL dislocation were IOL exchange and IOL refixation. The usual treatment for in-the-bag dislocations were intraocular lens complex explantation with or without new IOL sulcus fixation. However, for out-of-the-bag IOL dislocation, several factors may influence the choice of old IOL refixation or new IOL exchange: the amount of capsule support, the position and degree of the zonular dialysis, and other factors. In this study, we found that 19 eyes were subjected to two IOL repositionings before the latest sutured scleral refixation. Part of the reason is that the IOL is placed in the sulcus; however, the capsular support is inadequate, leading to repeated IOL dislocation, especially downstairs dislocation. 
The lens capsule may be able to temporarily hold an IOL in the sulcus but not enough for permanent fixation. We suggest that once out-of-the-bag IOL dislocation occurs, the old IOL should be removed, and a new, larger diameter optic IOL should be fixed to the sulcus. The three-piece IOL is not recommended to be used, for the rigid haptics of a threepiece IOL may aggravate further damage to the already weak lens zonule. Moreover, with the advancement of technology, numerous surgical techniques for scleral fixation of dislocated IOL have been developed, for example, an innovative scleral pockets technique for intrascleral fixation of IOL [16], which is conducive to better positioning of IOL.

\section{Conclusion}

In conclusion, the most common IOL dislocation is out-ofthe-bag dislocation; the most common risk factors include trauma, high myopia, and eyes undergoing pars plana vitrectomy; a minimum follow-up of 5 years is suggested for eyes predisposed for undergoing cataract surgery.

\section{Data Availability}

The data used to support the findings of this study are available from the corresponding author upon request.

\section{Disclosure}

Qi Fan and Xiaoyan Han are co-first authors. Jin Yang and Yi $\mathrm{Lu}$ are co-corresponding authors.

\section{Conflicts of Interest}

The authors declare that there are no conflicts of interest regarding the publication of this paper.

\section{Authors' Contributions}

Jin Yang and Yi Lu designed the research; Xiaoyan Han, Lei Cai, and Xiaodi Qiu collected the data; and Qi Fan and Xiangjia Zhu analyzed the data. Qi Fan and Xiaoyan Han cowrote the manuscript. Jin Yang and Yi Lu critically revised the manuscript. Qi Fan and Xiaoyan Han contributed equally to the writing of the manuscript. Jin Yang and $\mathrm{Yi} \mathrm{Lu}$ contributed equally to the conduct of this research.

\section{Acknowledgments}

This study was presented as free paper in 32nd APACRS KYOTO 2019. This work was supported by the National Natural Science Foundation of the People's Republic of China (NSFC) (Grants nos. 81600718 and 81670835), Scientific Research Project of Shanghai Health Bureau (Grant No. 201740033), Scientific Research Program, Science and Technology Commission of Shanghai Municipality (Grant No. 16ZR405200) and the Shanghai High Myopia Study (Grant no. +NCT03062085).

\section{References}

[1] F. J. Ascaso, V. Huerva, and A. Grzybowski, "Epidemiology, etiology, and prevention of late IOL-capsular bag complex dislocation: review of the literature," Journal of Ophthalmology, vol. 2015, Article ID 805706, 7 pages, 2015.

[2] G. Jakobsson, M. Zetterberg, M. Lundström, U. Stenevi, R. Grenmark, and K. Sundelin, "Late dislocation of in-the-bag and out-of-the bag intraocular lenses: ocular and surgical characteristics and time to lens repositioning," Journal of Cataract \& Refractive Surgery, vol. 36, no. 10, pp. 1637-1644, 2010.

[3] H. V. Gimbel, G. P. Condon, T. Kohnen, R. J. Olson, and I. Halkiadakis, "Late in-the-bag intraocular lens dislocation: incidence, prevention, and management," Journal of Cataract \& Refractive Surgery, vol. 31, no. 11, pp. 2193-2204, 2005.

[4] W. J. Stark, A. E. Maumenee, M. Datiles et al., "Intraocular lenses: complications and visual results," Transactions of the American Ophthalmological Society, vol. 81, pp. 280-309, 1983.

[5] S. L. Pueringer, D. O. Hodge, and J. C. Erie, "Risk of late intraocular lens dislocation after cataract surgery, 1980-2009: a population-based study," American Journal of Ophthalmology, vol. 152, no. 4, pp. 618-623, 2011.

[6] K. Dabrowska-Kloda, T. Kloda, S. Boudiaf, G. Jakobsson, and U. Stenevi, "Incidence and risk factors of late in-the-bag intraocular lens dislocation: evaluation of 140 eyes between 1992 and 2012," Journal of Cataract \& Refractive Surgery, vol. 41, no. 7, pp. 1376-1382, 2015.

[7] P. Vazquez-Ferreiro, F. J. Carrera-Hueso, N. Fikri-Benbrahim, L. Barreiro-Rodriguez, M. Diaz-Rey, and M. A. R. Barrios, "Intraocular lens dislocation in pseudoexfoliation: a systematic review and meta-analysis," Acta Ophthalmologica, vol. 95, no. 3, pp. e164-e169, 2017.

[8] S. Ganesh, P. Sen, and H. Sharma, "Late dislocation of in-thebag intraocular lenses in uveitic eyes: an analysis of management and complications," Indian Journal of Ophthalmology, vol. 65, no. 2, pp. 148-154, 2017.

[9] S. Masket, B. B. Ceran, and N. R. Fram, "Spontaneous dislocation of posterior chamber intraocular lenses (PC IOLs) in patients with retinitis pigmentosa-case series," Saudi Journal of Ophthalmology, vol. 26, no. 1, pp. 61-65, 2012.

[10] M. Matsumoto, K. Yamada, M. Uematsu et al., "Spontaneous dislocation of in-the-bag intraocular lens primarily in cases with prior vitrectomy," European Journal of Ophthalmology, vol. 22, no. 3, pp. 363-367, 2012.

[11] L. Werner, B. Zaugg, T. Neuhann, M. Burrow, and M. Tetz, "In-the-bag capsular tension ring and intraocular lens subluxation or dislocation," Ophthalmology, vol. 119, no. 2, pp. 266-271, 2012.

[12] K. Hayashi, A. Hirata, and H. Hayashi, "Possible predisposing factors for in-the-bag and out-of-the-bag intraocular lens dislocation and outcomes of intraocular lens exchange surgery," Ophthalmology, vol. 114, no. 5, pp. 969-975, 2007.

[13] R. Lorente, V. de Rojas, P. V. de Parga et al., "Management of late spontaneous in-the-bag intraocular lens dislocation: retrospective analysis of 45 cases," Journal of Cataract \& Refractive Surgery, vol. 36, no. 8, pp. 1270-1282, 2010.

[14] K. Hayashi, S. Ogawa, S.-i. Manabe, A. Hirata, and K. Yoshimura, "A classification system of intraocular lens dislocation sites under operating microscopy, and the surgical techniques and outcomes of exchange surgery," Graefes Archive for Clinical and Experimental Ophthalmology, vol. 254, no. 3, pp. 505-513, 2016. 
[15] B. J. Shingleton, Y. Yang, and M. W. O’Donoghue, “Management and outcomes of intraocular lens dislocation in patients with pseudoexfoliation," Journal of Cataract \& Refractive Surgery, vol. 39, no. 7, pp. 984-993, 2013.

[16] M. Postorino, A. Meduri, L. Inferrera et al., "Scleral pockets for an innovative technique of intrascleral fixation of intraocular lens," European Journal of Ophthalmology, vol. 2019, Article ID 112067211986601, 2019. 